\title{
Surgical management of moderate ischemic mitral regurgitation at the time of coronary artery bypass grafting remains controversial
}

\author{
Robert E. Michler, MD
}

\footnotetext{
From the Departments of Surgery and Cardiothoracic and Vascular Surgery, Montefiore Medical Center, Albert Einstein College of Medicine, New York, NY.

Received for publication April 11, 2018; revisions received April 11, 2018; accepted for publication May 1, 2018; available ahead of print June 27, 2018.

Address for reprints: Robert E. Michler, MD, Department of Cardiothoracic and Vascular Surgery, Montefiore Medical Center, Albert Einstein College of Medicine, New York, NY 10467 (E-mail: rmichler@montefiore. org).

J Thorac Cardiovasc Surg 2018;156:1498-500 $0022-5223 / \$ 36.00$

Copyright (c) 2018 by The American Association for Thoracic Surgery https://doi.org/10.1016/j.jtcvs.2018.05.060
}

Ischemic mitral regurgitation (IMR) is a consequence of regional wall motion abnormalities induced by myocardial ischemia or infarction. ${ }^{1}$ Adverse left ventricular (LV) remodeling develops in approximately $50 \%$ of patients after a myocardial infarction, and moderate mitral regurgitation (MR) occurs in more than $10 \%$ of patients. ${ }^{2,3}$ The presence of any degree of IMR discriminates patients with LV dysfunction who have a higher mortality risk than those without IMR, regardless of the treatment.

MR results from a combination of regional wall motion abnormalities, papillary muscle displacement, leaflet tethering, reduced closing forces, and annular dilatation. ${ }^{1}$ The mitral valve leaflets appear normal to the surgeon. Most patients have multivessel coronary artery disease requiring revascularization, so surgeons must consider whether to add a mitral valve repair procedure to coronary artery bypass grafting (CABG) in patients with moderate IMR.

The appropriate surgical management of moderate IMR at the time of $\mathrm{CABG}$ remains controversial. Some experts advocate revascularization alone for moderate IMR, expecting improvements in regional and global LV function and geometry after CABG to lead to a reduction in MR. ${ }^{4,5}$ Others support restrictive mitral annuloplasty (RMA) repair at the time of $\mathrm{CABG}$ to address the IMR more directly, expecting to prevent further adverse remodeling

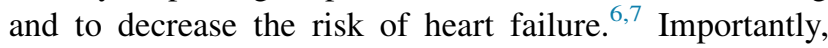
the addition of a mitral valve procedure to CABG surgery necessitates open heart exposure and is associated with longer durations of aortic crossclamping and cardiopulmonary bypass, which can increase perioperative risk. $^{8}$

Numerous observational, nonrandomized, single-center experiences are informative, but they suffer from the limitations of single-center experiences, study design, nonrigorous definition of the degree of $\mathrm{MR}$, and the inclusion in the analysis of patients with both moderate

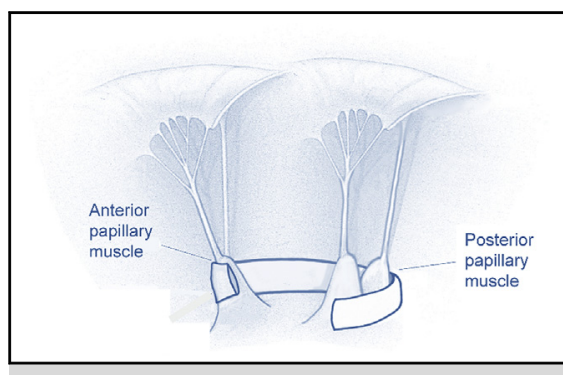

Papillary muscle approximation reduces LV endsystolic dimension and leaflet tethering. Reprinted with permission.

Central Message

A reparative procedure is recommended for select cases of moderate ischemic MR: LV scar or aneurysm/dyskinesia, large ventricles, and poor coronary targets because of a low likelihood to improve LVEF.

See Editorial Commentary page 1501.

and severe degrees of MR. Three recent randomized clinical trials of moderate IMR with well-defined criteria for the measurement of MR have compared CABG alone versus CABG plus RMA. ${ }^{6-8}$

Both Fattouch and colleagues ${ }^{6}$ and the Randomized Ischemic Mitral Evaluation (RIME) investigators ${ }^{7}$ demonstrated that the addition of RMA resulted in improvements in LV reverse remodeling, LV ejection fraction, New York Heart Association functional class, and MR grade, but not in survival. Fattouch and colleagues ${ }^{6}$ randomly assigned 102 patients to undergo $\mathrm{CABG}$ alone or $\mathrm{CABG}$ plus RMA. They demonstrated that the addition of RMA significantly reduced LV end-systolic dimension (LVESd). The RIME trial ${ }^{7}$ randomly assigned 73 patients to undergo CABG alone or CABG plus RMA and demonstrated a $28 \%$ reduction in $\mathrm{LV}$ end-systolic volume index (LVESVI) relative to baseline (mean baseline, $78 \mathrm{~mL} / \mathrm{m}^{2}$ ).

The National Heart, Lung, and Blood Institute-sponsored Cardiothoracic Surgical Trials Network (CTSN) more recently conducted a multicenter randomized trial $^{8,9}$ comparing CABG alone with CABG plus RMA in 301 patients with moderate IMR. RMA resulted in a significant reduction relative to $\mathrm{CABG}$ alone in prevalences of $\mathrm{MR}$ at 1 and 2 years, with no progression to severe MR in the 
RMA group. ${ }^{9}$ There were no differences between the 2 groups in the degree of LV reverse remodeling (LVESVI), New York Heart Association functional class, LV ejection fraction, survival, or major adverse cardiac and cerebrovascular events at 1 and 2 years. RMA was associated with a longer hospital stay after surgery, a higher incidence of post-operative supraventricular arrhythmias and more postoperative neurologic events, (which included metabolic encephalopathy, seizures, transient ischemic attack, and stroke).

It is well established that improvements in global and regional wall motion as well as reverse $\mathrm{LV}$ remodeling after CABG alone are indicative of viable myocardium. ${ }^{5,10}$ For example, Penicka and colleagues ${ }^{4}$ found that in a series of patients with moderate IMR who underwent CABG alone, resolution of MR after surgery was associated with more viable segments and less LV dyssynchrony at baseline.

In the CTSN trial, irrespective of treatment arm, patients with resolution of IMR had greater reverse remodeling and better wall motion scores than those who did not. ${ }^{9}$ This latter point is noteworthy, because it provides evidence that many patients in both arms of the CTSN trial must have had viable myocardium and not LV scar tissue, which resulted in an improvement in wall motion, a reduction in LV size, and less MR with just the revascularization procedure.

There are multiple comparative points to emphasize regarding these 3 clinical trials. First, the sample sizes differed greatly; the CTSN trial enrolled more than 3 times the number of patients in the other trials. Second, the 3 clinical trials used different primary end points. Third, the analytic approaches were different, with the CTSN trial counting patients who died as treatment failures in the primary end point analysis, whereas the other trials used a survivor analysis. Fourth, the CTSN trial had significantly lower rates of baseline previous MI and possibly less LV scar tissue. Fifth, and perhaps most importantly, baseline LV size was significantly larger in the trial of Fattouch and colleagues ${ }^{6}$ and the RIME trial $^{7}$ than in the CTSN trial. This fact alone may have favored patients who received a restrictive annuloplasty, especially if more scar tissue was present, because CABG alone in these patients would be less likely to result in an improvement in LV wall motion and reduced LV size, precisely those factors that lead to less MR.

Not surprisingly, it is in patients with LV scar tissue for whom CABG alone is less likely to provide benefit and precisely those patients who may need the addition of RMA to reduce IMR. In the RIME trial, ${ }^{7}$ a $28 \%$ reduction in LV size from baseline (mean baseline, $78 \mathrm{~mL} / \mathrm{m}^{2}$ ) was achieved with CABG plus RMA, whereas in the CTSN trial, those patients who received the combined procedure saw only a $9 \%$ reduction in LV size from baseline (mean, baseline $57 \mathrm{ml} / \mathrm{m}^{2}$ ). To underscore this important point, patients in the CTSN trial had smaller ventricles at baseline and, as the evidence suggests, more viable myocardiumprecisely the clinical substrate that is likely to benefit most from CABG alone.

Surgical decision making for patients with moderate IMR therefore could be enhanced by preoperative identification of those patients most likely to have an improvement in regional wall motion and global $\mathrm{LV}$ function from $\mathrm{CABG}$ alone. Not one of these 3 clinical trials required preoperative evaluation of myocardial viability. It is known that viability assessment can predict the effectiveness of revascularization in specific patient populations, and one should therefore consider it in this setting. ${ }^{11}$ Cardiac magnetic resonance imaging with gadolinium hyperenhancement is an appropriate tool when viability imaging results are equivocal or LV scar tissue is suspected.

Individual treatment decisions require balancing the risks of adverse perioperative events against the benefits of a lower incidence of postoperative MR. Effective revascularization, as reflected in improved regional and global LV function, plays an important role in reducing MR, independent of restrictive annuloplasty repair. In other clinical settings, the anticipated low likelihood of generating significant functional improvement and reverse remodeling from CABG should lead to the performance of a mitral valve reparative procedure. Such circumstances include patients with documented scar tissue or basal aneurysm or dyskinesia in the inferoposterior lateral $\mathrm{LV}$, large ventricles (LVESVI $>60 \mathrm{~mL} / \mathrm{m}^{2}$ and left ventricular end diastolic diameter $>50 \mathrm{~mm}$ ) and poor coronary targets in the circumflex and right coronary distributions because of the reduced likelihood that revascularization will provide significant enhancement of LV contractility and LV reverse remodeling.

Recent post hoc analysis by the CTSN authors ${ }^{12}$ has highlighted the importance of evaluating the ratio of LVESd to prosthetic annuloplasty ring size in predicting the risk of persistent or recurrent MR after surgery in patients with moderate and severe IMR. The CTSN authors ${ }^{12}$ noted that a LVESd/ring size ratio of at least 2 was associated (multivariate analysis) with increased risk of persistent or recurrent MR. Therefore, insertion of too small of a prosthetic ring could increase this ratio and lead to persistent MR or even mitral stenosis. In my judgment, patients with an enlarged ventricle (especially those with scar tissue, dyskinesia, or a basal aneurysm) in whom mitral repair is feasible should therefore receive a ventricular procedure to surgically reduce LVESd together with a restrictive annuloplasty procedure. A papillary muscle approximation has been used in clinical trials of severe IMR to produce excellent long-term freedom from recurrent or persistent IMR. ${ }^{13}$ A papillary muscle approximation procedure mechanically reduces the LVESd and has the potential to reduce leaflet tethering, thus producing a lower ratio of LVESd to ring size and a realistic expectation of reducing MR. 
My current decision algorithm for managing moderate IMR focuses on 5 preoperative factors that help determine the surgical plan. In conjunction with echocardiography and cardiac catheterization, cardiac magnetic resonance imaging can complete a comprehensive analysis of these factors.

1. Severity of MR

2. Severity of LV dysfunction

3. Severity of LV remodeling (LVESVI)

4. Presence and extent of LV scar tissue

5. Quality and distribution of the left circumflex and right coronary circulations

Whether RMA repair together with CABG will predictably benefit patients with an enlarged LV or with baseline inferoposterior lateral wall motion abnormalities considered to represent scar tissue remains unknown. Until such evidence is available, RMA and papillary muscle approximation should be considered an essential part of the surgical armamentarium for patients with moderate IMR.

\section{Conflict of Interest Statement}

Author has nothing to disclose with regard to commercial support.

\section{References}

1. Grigioni F, Detaint D, Avierinos JF, Scott C, Tajik J, Enriquez-Sarano M Contribution of ischemic mitral regurgitation to congestive heart failure after myocardial infarction. J Am Coll Cardiol. 2005;45:260-7.

2. Bursi F, Enriquez-Sarano M, Nkomo VT, Jacobsen SJ, Weston SA, Meverden RA, et al. Heart failure and death after myocardial infarction in the community: the emerging role of mitral regurgitation. Circulation. 2005;111: 295-301.

3. Pérez de Isla L, Zamorano J, Quezada M, Almería C, Rodrigo JL, Serra V, et al. Functional mitral regurgitation after a first non-ST-segment elevation acute coronary syndrome: contribution to congestive heart failure. Eur Heart $J$ 2007:28:2866-72.

4. Penicka M, Linkova H, Lang O, Fojt R, Kocka V, Vanderheyden M, et al. Predictors of improvement of unrepaired moderate ischemic mitral regurgitation in patients undergoing elective isolated coronary artery bypass graft surgery. Circulation. 2009;120:1474-81.

5. Roshanali F, Mandegar MH, Yousefnia MA, Alaeddini F, Wann S. Low-dose dobutamine stress echocardiography to predict reversibility of mitral regurgitation with CABG. Echocardiography. 2006;23:31-7.

6. Fattouch K, Guccione F, Sampognaro R, Panzarella G, Corrado E, Navarra E, et al. Point: efficacy of adding mitral valve restrictive annuloplasty to coronary artery bypass grafting in patients with moderate ischemic mitral valve regurgitation: a randomized trial. J Thorac Cardiovasc Surg. 2009;138: 278-85.

7. Chan KM, Punjabi PP, Flather M, Wage R, Symmonds K, Roussin I, et al; RIME Investigators. Coronary artery bypass surgery with or without mitral valve annuloplasty in moderate functional ischemic mitral regurgitation: final results of the randomized ischemic mitral evaluation (RIME) trial. Circulation. 2012; 126:2502-10.

8. Smith PK, Puskas JD, Ascheim DD, Voisine P, Gelijns AC, Moskowitz AJ, et al; Cardiothoracic Surgical Trials Network Investigators. Surgical treatment of moderate ischemic mitral regurgitation. N Engl J Med. 2014;371:2178-88.

9. Michler RE, Smith PK, Parides MK, Ailawadi G, Thourani V, Moskowitz AJ, et al; CTSN. Two-year outcomes of surgical treatment of moderate ischemic mitral regurgitation. N Engl J Med. 2016;374:1932-41.

10. Sundt TM. Surgery for ischemic mitral regurgitation. $N$ Engl J Med. 2014;371: 2228-9.

11. Meluzín J, Cerný J, Frélich M, Stetka F, Spinarová L, Popelová J, et al. Investigators of this multicenter study... Prognostic value of the amount of dysfunctional but viable myocardium in revascularized patients with coronary artery disease and left ventricular dysfunction. J Am Coll Cardiol. 1998;32: 912-20.

12. Capoulade R, Zeng X, Overbey JR, Ailawadi G, Alexander JH, Ascheim D, et al; Cardiothoracic Surgical Trials Network (CTSN) Investigators. Impact of left ventricular to mitral valve ring mismatch on recurrent ischemic mitral regurgitation after ring annuloplasty. Circulation. 2016;134:1247-56.

13. Nappi F, Lusini M, Spadaccio C, Nenna A, Covino E, Acar C, et al. Papillary muscle approximation versus restrictive annuloplasty alone for severe ischemic mitral regurgitation: a randomized trial. J Am Coll Cardiol. 2016;24:2334-46.

14. Nappi F, Spadaccio C, Nenna A, Lusini M, Fraldi M, Acar C, et al. Is subvalvular repair worthwhile in severe ischemic mitral regurgitation? Subanalysis of the Papillary Muscle Approximation trial. J Thorac Cardiovasc Surg. 2017;153: 286-95.e2. 